\title{
Normalization Framework for Vulnerability Risk Management in Cloud
}

\author{
Vida Ahmadi \\ Dept. of Computer Science \\ Blekinge Institute of Technology \\ City Network International $A B$ \\ Karlskrona, Sweden \\ email: vida.ahmadi@citynetwork.eu
}

\author{
Patrik Arlos \\ Dept. of Computer Science \\ Blekinge Institute of Technology \\ Karlskrona, Sweden \\ email: patrik.arlos@bth.se
}

\author{
Emiliano Casalicchio \\ Dept. of Computer Science \\ Sapienza University of Rome, Italy \\ Blekinge Institute of Technology, Sweden \\ email: casalicchio@di.uniroma1.it
}

\begin{abstract}
Vulnerability Risk Management (VRM) is a critical element in cloud security that directly impacts cloud providers' security assurance levels. Today, VRM is a challenging process because the dramatic increase of known vulnerabilities $(+26 \%$ in the last five years), and because it is even more dependent on the organization's context. Moreover, the vulnerability's severity score depends on the Vulnerability Database (VD) selected as a reference in VRM. All these factors introduce a new challenge for security specialists in evaluating and patching the vulnerabilities. This study provides a framework to improve the classification and evaluation phases in vulnerability risk management while using multiple vulnerability databases as a reference. Our solution normalizes the severity score of each vulnerability based on the selected security assurance level. The results of our study highlighted the role of the vulnerability databases in patch prioritization, showing the advantage of using multiple VDs.

Index Terms - Vulnerability, Cloud security, Risk assessment
\end{abstract}

\section{INTRODUCTION}

Vulnerability identification, classification, and evaluation are listed as the vital criteria in the Cloud Risk Assessment models [1]. Vulnerability Risk Management (VRM) is the methodology used to identify, classify, evaluate, and remediate vulnerabilities. VRM usually involves operations that require IT security specialists intervention which is time-consuming and costly. Security specialists use vulnerability scanning tools (e.g., OpenVAS, Nexpose, and Nessus are examples of vulnerability scanners) to identify known system vulnerabilities. Vulnerability scanners rely on Vulnerability Databases (VDs), which are repositories that list the vulnerabilities, each having a unique identification number, a severity score, and patch instructions (used in the remediation phase). Because VDs are maintained by different institutions they use different severity scoring systems and could cover different sets of vulnerabilities. Hence, for security experts, is challenging to select the appropriate VD and eventually they need to rely multiple VDs. In this paper, we address the aforementioned problem. We consider vulnerabilities that are documented in the Common Vulnerabilities and Exposures (CVE) referencing system and are identified by a unique CVE-ID. Some of the VDs, such as the National Vulnerability Database (NVD), use the Common Vulnerability Scoring System (CVSS) framework to rank the severity of the CVE-ID, and others develop their own scoring system frameworks. Therefore, the same CVE-ID could have different severity score depending on what VD is used. For instance, CVE-2020-8130 has a severity score "8.1" in NVD [2], "Medium" in USN [3], "9" in DSA [4], and "Moderate" in RHSA [5]. Additionally, the time when VDs publish their ratings also differ, some are fast, some take a bit more time. The patching instructions and remediation strategy may also differ between the VDs.

Hence the variety of scoring systems available, which results in different severity scores and remediation instructions, could affect the outcome of the VRM process and, as the authors in [6]-[8] point out, it legitimates the following research questions:

1) Is one VD enough to use in a VRM?

2) What criteria should be considered to select the reference VD in VRM?

3) What would we gain by using multiple VDs in a VRM?

Answering the above research question is important for two reasons. Firstly, VRM is key to guarantee a desired security assurance level in cloud services as described in the European Cybersecurity Certification Scheme for Cloud Services (EUCS) [9] and in the NIST guidelines for Cloud security [10], [11]. Therefore, the security expert should be provided with guidelines on which VDs to use and if and how the VD selection impact the results of the vulnerability analysis and patch prioritization. Secondly, the number of published CVE-IDs has increased enormously during the last five years, from 6447 in 2016 to 18356 in 2020 [2], resulting in a wider differentiation among the VDs and introducing additional challenges when VRM is applied to complex system such as IaaS [12].

The main contributions of this study are outlined in what follows. Firstly, we proposed a VDs Normalization Framework (VDNF) that could be integrated in the vulnerability management process. The VDNF is a refinement and implementation of what envisioned in [13]. VDNF is intended as a tool to improve the classification and evaluation phases in VRM while dealing with multiple VDs as a source. For example, VDNF could be used by IaaS security specialist dealing with the challenge of selecting the VDs more appropriate for the specific context of 
their organization [8].

Secondly, we implemented our VDNF and we validated the VDNF on a large set of sampled CVE-IDs. We chose the CVE-IDs that affected Ubuntu's releases from 2017 to 2020 as Ubuntu is the most used Linux distribution in Cloud computing [14]. We collected the severity score of the sampled CVE-IDs from NVD, USN, and DSA databases to cover multiple VDs.

Finally, we analyzed the distribution of severity scores in the different VDs and we analyzed the impact of the normalization framework on generalizing the severity score of the various VDs. Results confirm that using a single VD do not allow to properly analyze vulnerabilities in a complex organization context. Indeed, VDs normalization allows to cover a larger number of CVE-IDs (e.g., USN does not consider a not negligible set of CVE-IDs that are considered in DSA and NVD). Hence, in complex contexts VRM benefits of VDs normalization.

This paper is organised as in what follows. Related works are analyzed in Section II. Section III firstly describes the issues in current approaches to VRM and then it describes how the proposed solution could improve VRM. Section V presents the normalisation framework and our sampling method. The analyses of the result of our solution are presented in Section VI. Finally, Section VII concludes the paper.

\section{RELATED WORKS}

As mentioned before, the research community have recently raised the challenges introduced by the existence of multiple VDs. For example, the authors in [6] provided a comprehensive survey on the vulnerability scanning tools and databases to support security specialists in selecting the right vulnerability scanning tools and VDs. They point out the need for orchestration in vulnerability scanning tools for obtaining the highest vulnerability coverage. However, their study is limited to the vulnerability scanning tools and VDs based using the vulnerability taxonomy defined by the Open Web Application Security Project (OWASP) [15]. Moreover their study covered only the web application vulnerability detection tools [6]. The study by Dey et al. [7] concluded that patch prioritisation based on the single metric such as severity score from one VD is not an optimal patch policy.

Furthermore, the risk and impact of exploiting vulnerabilities depend on the organization's assets, security requirements, and security policies. Thus, the exploitation risk should be evaluated in the context of the organization [8]. Therefore, security specialists need to define their asset-based criteria using multiple VDs and vulnerability scanning tools. The lack of proper classification of vulnerabilities in VDs leads to some knowledge-based VDs where vulnerabilities are divided into multiple classes based on the impact of vulnerabilities' exploit [16]. Unfortunately, such VDs only use one VD as a source of truth for their classification, which does not provide the proper coverage. The state of vulnerability in 2020 shows only $84 \%$ of the vulnerabilities are registered in CVE [17] registry with an assigned CVE-ID. However, most of those unregistered vulnerabilities are related to the software patched by the software community. The security specialist should check the software's status in their organization besides the VDs, which is a time-consuming task [18].

The authors in [19] described the time factor corresponding to the vulnerability risk management and patch management. They proposed the vulnerability management center where the data collected from organization inventory and NVD database are integrated with the vulnerability scanner. Their paper aims to reduce the time of vulnerability risk management procedures. However, they used one generic VD as a reference, and calculated the environmental metrics based on the CVSS v2.0 standard. Such an approach required verifying the obtained score with the subject-specific VDs, which was missing in the paper.

From our literature study emerge that although the research community recognized the limitation of considering only a single VD in VRM, no work provide a clear answer to the research questions addressed by our paper.

\section{The Role of Vulnerability Databases IN VulNERABILITY RisK MANAGEMENT}

VDs are the repositories of publicly known vulnerabilities usually maintained by a community or organization with a specific area of interest, i.e. the subject-specific VDs. For instance, NIST National Vulnerability Database (NVD) hosts all published CVE-IDs to support the US Department of Commerce. Canonical hosts USN, a VD for tracking the vulnerabilities that affect Ubuntu releases. Debian maintains a VD containing the Debian Security Advisories (DSAs), i.e., security vulnerability that affects a Debian package. NVD is the largest VD available, it stores CVE-IDs record since 1988, and it is used as a reference VD in most of the vulnerability scanners. NVD applies the based score of the CVSS framework to rank the CVE-IDs, while Canonical developed their framework for ranking the CVE-IDs that affected their releases. The criteria and the method for calculating a severity score by Canonical are not publicly available. However, Canonical claims that their scoring system considers the impact of the vulnerability in their environments. DSA relies on NVD scores.

VRM consists of four phases: $i$ ) identification; $i i)$ classification and analysis; $i i i$ ) evaluation; and $i v$ ) remediation. The vulnerability identification phase and the vulnerability classification and analysis phase rely on VDs; phases iii) and $i v$ ) use the results from phase $i i)$. Hence the results of VRM are effected by the selected VD, as detailed in what follow.

The first phase identifies the system's vulnerability by using vulnerability scanners. Those tools report the detected vulnerabilities and a severity score. The severity score they report is calculated by the VD used by the selected tool, either by design or by configuration. That VD, may or may not use the criteria listed in the CVSS framework, to calculate the severity score. Therefore, the VD and its scoring system play a vital role in VRM.

The second phase is the classification and analysis of detected vulnerabilities. Most security specialists use the severity score 
to classify the vulnerabilities (e.g., detected CVE-ID with a critical severity score). Some experts use the type of exploit (e.g., detected CVE-ID that a remote attacker could exploit). The classification of the vulnerabilities impacted the evaluation for patch prioritization in the next phase. Which raises the question, which VD's severity score should consider?

The third phase is the evaluation of the impact that the vulnerabilities have, and define a patch priority. This requires in-depth knowledge about the security policy, probability of exploitation, and impact of the exploit on the system to plan a proper response and remediation. For example, the vulnerability that can exploit by the remote attacker have a higher risk than the one required local access; hence the first get a higher priority than the second.

Remediation is the last step in VRM, which patches the detected vulnerabilities, based on their priority. The remediation phase mostly relies on the software or hardware vendor (e.g., patch intel-microcode vulnerability should install the software's minimum safe version). However, some vulnerabilities might remain unpatched in a system due to the limited attack vector.

Our VRM approach relies on the organization's knowledge, to help security decision-makers analyze and evaluate vulnerabilities within the organization's context, rather than purely relying on severity scores. Organizational knowledge refers to system management documents, such as security policy, system configuration, asset inventory, and software inventory. A context-aware VRM mitigates the challenge with different severity scores from VDs, c.f. [13].

The proposed VRM approach encourages the selection of VD based on the organization's asset inventory. For instance, the Canonical severity score should use for patch prioritization in the organization with only the Ubuntu releases in asset inventory. For the organization with various vendors, multiple VDs should use as a reference. As we mentioned in Section I, some VDs provide a numerical score while the others give a qualitative score. Hence obtaining a single severity score for each CVE-ID is a new challenge in VRM. The proposed VRM approach introduce a normalization framework to support security experts and decision-makers in phases I and II.

\section{THE VDS NORMALISATION FRAMEWORK}

The Normalization framework proposes a method to obtain a single severity score for each CVE-ID by aggregating scores from multiple VDs. The framework offers three working modes, hereafter Vulnerability Management Mode (VMM): Basic, Standard, and Restrictive. The proposed VMM are inline with the three assurance levels proposed in the ENISA cybersecurity certification framework [9]. A Basic VMM is the minimum acceptable baseline for a VRM process to cover a system's vulnerability identification, and with a limited public exposure scope. A Standard VMM is suitable to serve a system with medium to a high security risk, while the Restrictive mode should be used for VRM in compliant systems (i.e., the particular system that should comply with the local or international regulatory and standards) and critical infrastructure. Hence, it should be easy to select a VMM meeting the required organizations' assurance level.

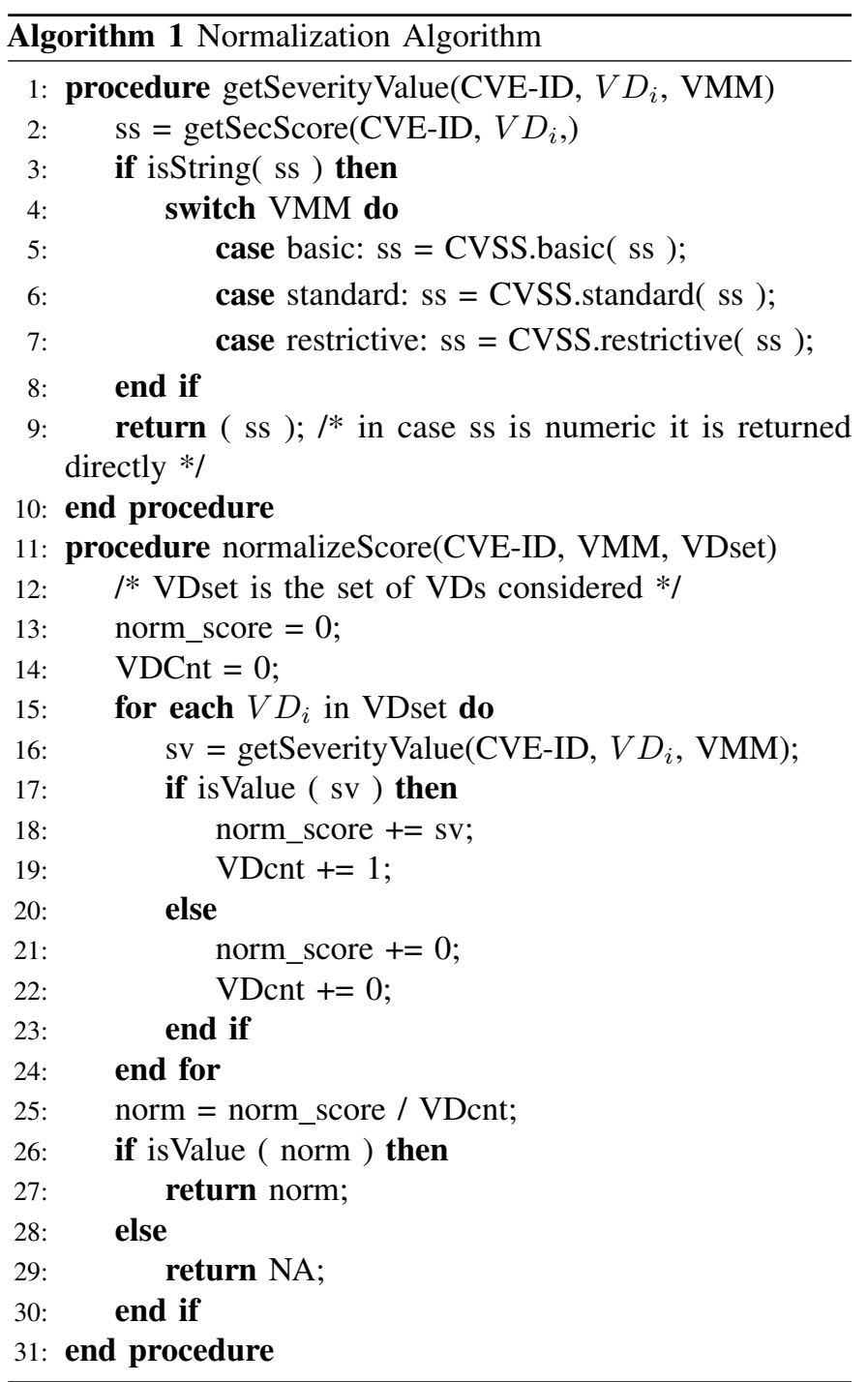

The core components of the normalization framework are described in Algorithm 1. Inputs to the normalization framework are: a list of CVE-IDs, a list of the desired VDs, and the VMM. The framework produces as output, a normalized severity score per CVE-ID.

The getSeverity Value procedure, obtains the severity score for the identified CVE-ID and selected VD. If the severity score ("ss") is qualitative, the framework converts ss to the numeric value based on the severity score range in Table I. For example if $s s=L o w$ and $V M M=$ standard, then $s s$ is turned into the value of 2 . If the severity score is numeric, it is not altered. The second procedure, normalizeScore, calculates the average severity score for the CVE-ID.

As each organization behind a VD operates differently, there are scenarios when some VDs might not have a score for all the published CVE-ID. Using the normalization framework, we can facilitate better coverage, as it relies on input from multiple VDs. In this way, reducing the probability of having 
TABLE I: Severity Score range in CVSS v3.x and the normalisation framework

\begin{tabular}{|c|c|c|c|c|}
\hline CVSS v3.x Score & Qualitative Rate & Basic VMM & Standard VMM & Restrictive VMM \\
\hline 0.0 & None & 0 & 0 & 0 \\
\hline $0.1-3.9$ & Low & 0.1 & 2 & 3.9 \\
\hline $4.0-6.9$ & Medium & 4.0 & 5.45 & 6.9 \\
\hline $7.0-8.9$ & High & 7.0 & 7.95 & 8.9 \\
\hline $9.0-10.0$ & Critical & 9.0 & 9.5 & 10.0 \\
\hline
\end{tabular}

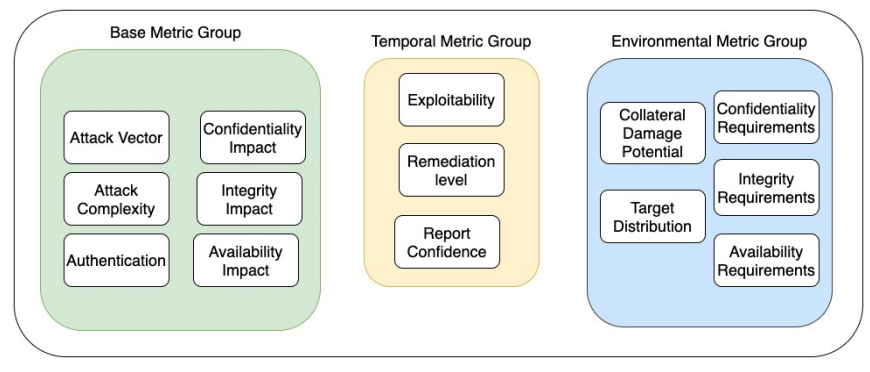

Fig. 1: CVSS v2.0 metrics [20]

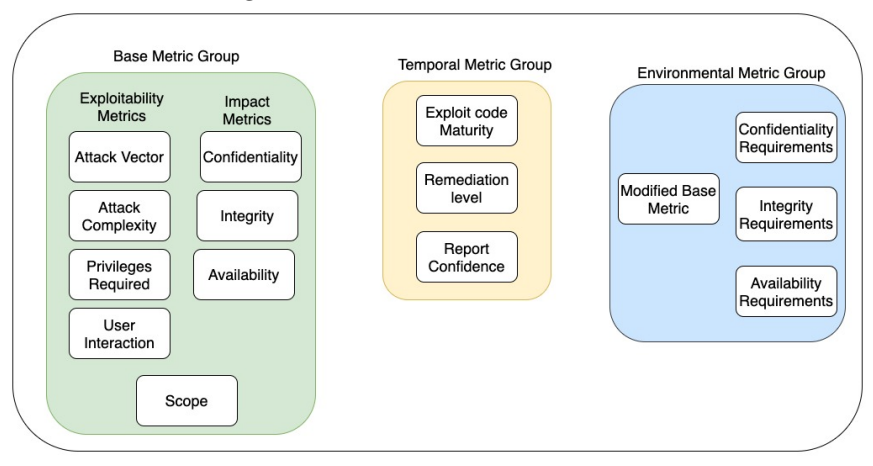

Fig. 2: CVSS v3.x metrics [21]

a CVE-ID without a score.

The normalization framework uses the CVSS v3.x (i.e. version 3 and above) scoring system [21] as a reference, when converting a qualitative score to a numeric value. We use CVSS v3.x because it provides a more accurate view of the security impact on the system by expanding the basic metrics group. For instance, the authentication metrics are divided into two exploit-ability metrics, the privileges required and the user interaction, to provide a better evaluation on authenticity impacts, c.f. Figure 1 and Figure 2. Furthermore, a scope metric has been added to the basic metrics, capturing whether a vulnerability in one component affects resources managed by one or multiple security authorities. In cloud computing, this scope plays a vital role as it addresses vulnerabilities in guest (virtual) entities that could compromise the host (Cloud infrastructure), i.e. hypervisor attacks [21].

The CVSS v2.0 and v3.x metrics are presented in Figure 1 and Figure 2. CVSS v3 provides a fine grain scale in a score by dividing the high range in CVSS v2.0 into two ranges 7.0-8.9 and 9.0-10.0. The new scale provides better visibility on vulnerabilities that have a higher risk of exploitation.
TABLE II: Registered CVE-IDs VS. Sample CVE-IDs

\begin{tabular}{|c|c|c|}
\hline Year & Registered CVE-ID & Sample CVE-ID \\
\hline 2017 & 14646 & 4043 \\
\hline 2018 & 16511 & 3179 \\
\hline 2019 & 17305 & 2952 \\
\hline 2020 & 18355 & 2387 \\
\hline
\end{tabular}

\section{VALIDATION CASE STUDY}

To validate the proposed normalization framework, we apply the VDNF to three VDs that use different scoring mechanisms:

- National Vulnerability Database, NVD

- Debian Security Advisories, DSA

- Ubuntu Security Notices, USN

We chose NVD as it calculates a base score for CVSS v2.0 and v3.x, and records both numeric and qualitative score for each CVE-ID. We picked DSA as it reports a qualitative score which relies on NVD score but does not clarified the version of CVSS. The last, USN provides a qualitative score where the criteria and calculation method is not available. Hence, the severity scores from DSA and USN are qualitative and need to be converted to a numeric value by the normalization procedure in Algorithm 1.

To validate the framework, we have selected CVE-IDs that has affected Ubuntu's releases from 2017 to 2020 . We collected the information for those CVE-IDs on February 4th, 2021 from the selected VDs. For each VD we recorded the CVEID, severity score, description of the vulnerability, date of publishing CVE-ID, and a reference link. The data (available in GitHub) ${ }^{1}$ was saved in a CSV file, one for each year. Table II presents the number of the registered CVE-IDs, and the number of sample CVE-IDs, per year.

We use a Python script to process the data and implement the normalization framework, described in Algorithm 1.

\section{ANALYSIS RESUlT}

First, we analyze the distribution of severity scores for each of VDs. Then, we compare the distributions between the VDs. Finally, we analyze the impact that the normalization framework has on the distribution of severity scores.

\section{A. Distribution of Severity Scores}

1) USN: The severity score is reported as a priority in USN VD. Hence, severity score none is ranked negligible in USN. The majority of the CVE-IDs have a medium score, and only three CVE-IDs ranked critical in our samples space. The NA in the Figures 3 to 6 refers to the number of CVE-ID that does

\footnotetext{
${ }^{1}$ DVNF data repository https://github.com/vidaAhmadi/sample-CVE
} 


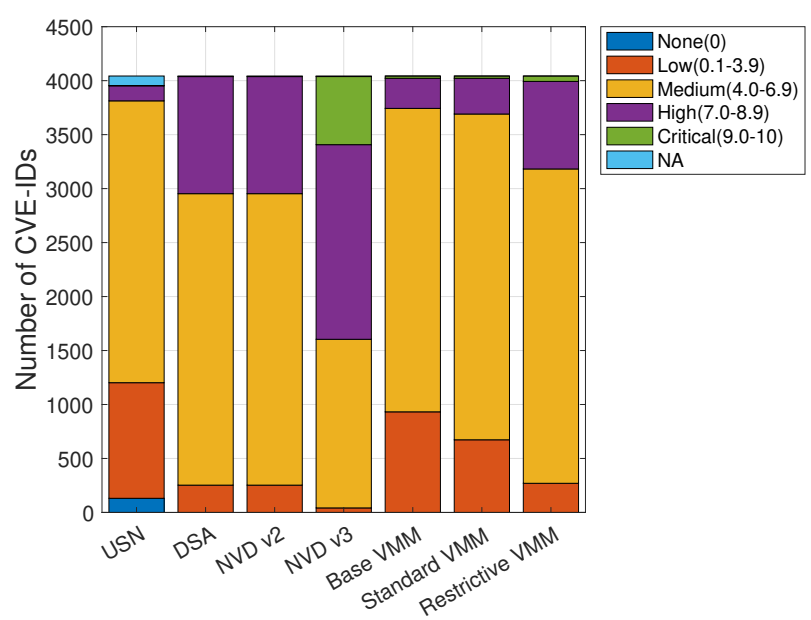

Fig. 3: Distribution of the severity scores in VDs and normalisation framework in 2017.

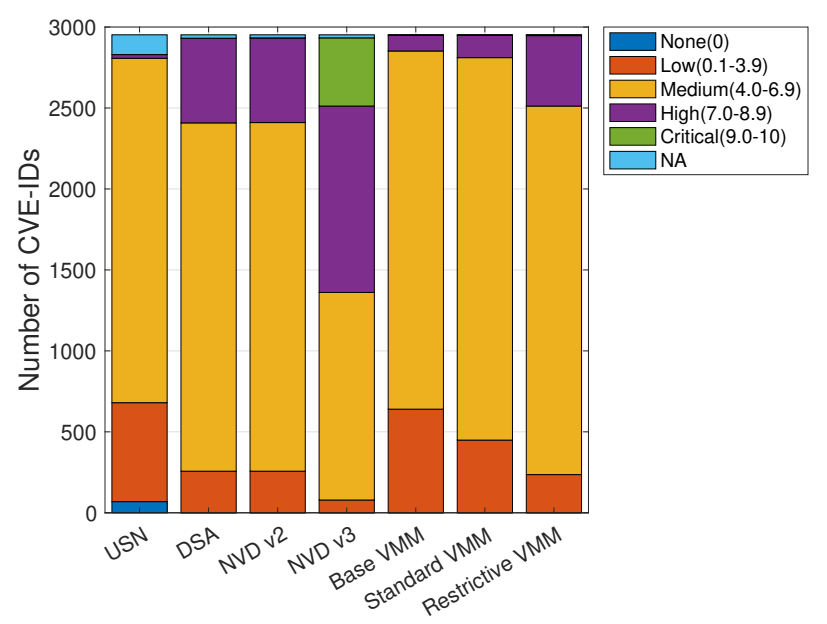

Fig. 5: Distribution of the severity scores in VDs and normalisation framework in 2019.

not have any score. USN has the highest number of NA in our samples. Furthermore, the number of registered CVE-IDs that affected Ubuntu releases decreased by $15.2 \%$ from 2017 to 2020 as shown in Table II.

2) DSA: Our result shows that DSA scores matches quite well with NVD v2. There is no critical score in our sample CVE-IDs, this proves that the scores are calculated with CVSS v2.0, or similar, because the critical range was introduced in CVSS v3.x. Furthermore, We did not find any evidence of independently calculated severity scores in the DSA VD or any reference to the framework used for calculating scores.

3) NVD: NVD uses the CVSS framework to calculate the severity score mentioned in its general documentation ${ }^{2}$. Most of the CVE-IDs have a severity score, from both CVSS v2.0 and v3.x, since 2016. The NVD score consists of the base metric group only, while the temporal metric and environment

${ }^{2}$ NVD documentation https://nvd.nist.gov/general

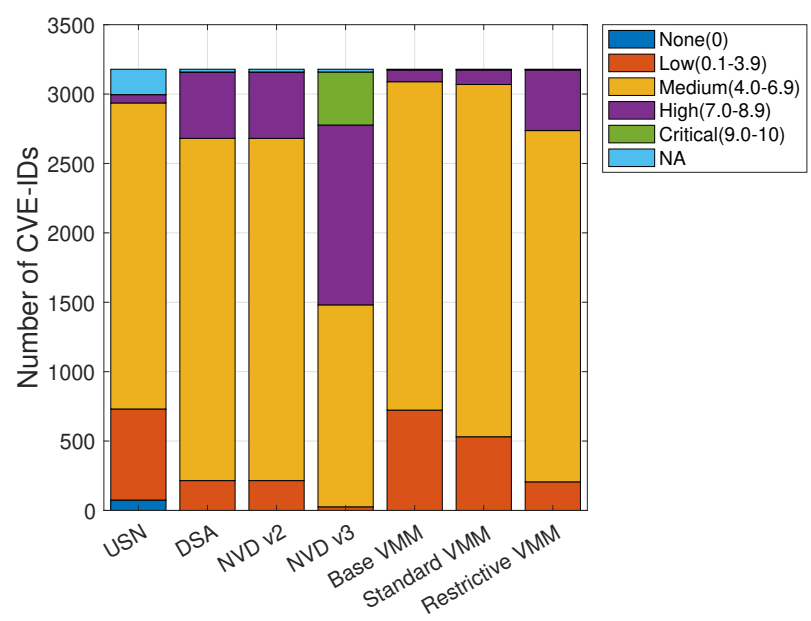

Fig. 4: Distribution of severity scores in VDs and normalisation framework in 2018.

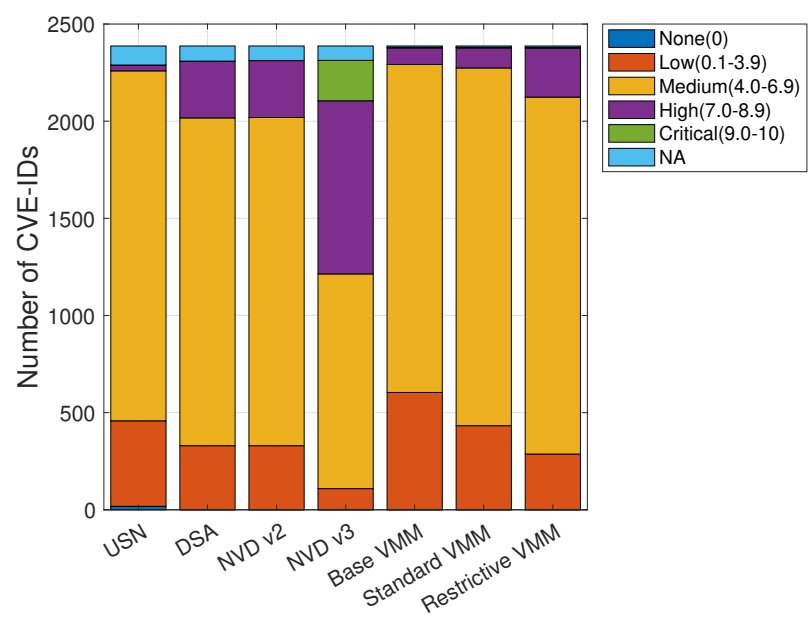

Fig. 6: Distribution of the severity scores in VDs and normalisation framework in 2020 .

metric are excluded in the value. Therefore, we consider NVD as a general-purpose VD.

Figures 3 to 6 visualized the NVD has the lowest number of NA in each year comparing with the other two VDs. We also see most CVE-IDs in NVD v2 are in the medium range. Furthermore, NVD reported the least number of CVE-IDs in the low range per year in our samples.

\section{B. Comparison of Distributions}

We selected USN and DSA as they are product-specific VDs. Ubuntu and Debian are both Linux distributions, one can expect a similar severity score in those databases. Furthermore, Ubuntu is built on-top of the Debian distribution. However, Table III and Figures 3 to 6 show no similarity between severity scores in DSA and USN. Nevertheless, the severity score reported by NVD v2 is similar to the one reported by DSA. Another finding is that the range None only exists in USN. 
TABLE III: Severity Score distribution in VDs and the normalisation framework 2017-2020

\begin{tabular}{|c|c|c|c|c|c|c|c|c|}
\hline & & \multicolumn{4}{|c|}{ VD } & \multicolumn{3}{|c|}{ VMM } \\
\hline Year & Score & USN & DSA & NVD CVSS v2.0 & NVD CVSS v3.x & Basic & Standard & Restrictive \\
\hline \multirow{6}{*}{2017} & 0.0 (None) & 131 & 0 & 0 & 0 & 0 & 0 & 0 \\
\hline & $0.1-3.9$ (Low) & 1071 & 253 & 253 & 42 & 932 & 673 & 270 \\
\hline & 4.0-6.9 (Medium) & 2611 & 2700 & 2700 & 1562 & 2811 & 3018 & 2912 \\
\hline & 7.0-8.9 (High) & 139 & 1085 & 1085 & 1803 & 279 & 331 & 811 \\
\hline & $9.0-10.0$ (Critical) & 3 & 0 & 0 & 631 & 21 & 21 & 50 \\
\hline & Not Available & 88 & 5 & 5 & 5 & 0 & 0 & 0 \\
\hline \multirow{6}{*}{2018} & 0.0 (None) & 75 & 0 & 0 & 0 & 0 & 0 & 0 \\
\hline & $0.1-3.9$ (Low) & 656 & 215 & 215 & 26 & 723 & 531 & 206 \\
\hline & 4.0-6.9 (Medium) & 2205 & 2466 & 2466 & 1455 & 2366 & 2538 & 2531 \\
\hline & 7.0-8.9 (High) & 60 & 478 & 478 & 1296 & 85 & 105 & 437 \\
\hline & 9.0-10.0 (Critical) & 0 & 0 & 0 & 382 & 3 & 3 & 3 \\
\hline & Not Available & 183 & 20 & 20 & 20 & 2 & 2 & 2 \\
\hline \multirow{6}{*}{2019} & 0.0 (None) & 69 & 0 & 0 & 0 & 0 & 0 & 0 \\
\hline & $0.1-3.9$ (Low) & 611 & 257 & 257 & 79 & 640 & 449 & 236 \\
\hline & 4.0-6.9 (Medium) & 2127 & 2151 & 2153 & 1282 & 2212 & 2362 & 2276 \\
\hline & 7.0-8.9 (High) & 23 & 523 & 523 & 1151 & 96 & 137 & 435 \\
\hline & $9.0-10.0$ (Critical) & 0 & 0 & 0 & 421 & 4 & 4 & 5 \\
\hline & Not Available & 122 & 21 & 19 & 19 & 0 & 0 & 0 \\
\hline \multirow{6}{*}{2020} & 0.0 (None) & 18 & 0 & 0 & 0 & 0 & 0 & 0 \\
\hline & $0.1-3.9$ (Low) & 440 & 330 & 330 & 109 & 604 & 433 & 287 \\
\hline & 4.0-6.9 (Medium) & 1801 & 1687 & 1689 & 1105 & 1688 & 1841 & 1837 \\
\hline & 7.0-8.9 (High) & 30 & 292 & 293 & 891 & 85 & 103 & 252 \\
\hline & 9.0-10.0 (Critical) & 0 & 0 & 0 & 208 & 3 & 3 & 4 \\
\hline & Not Available & 98 & 78 & 75 & 74 & 7 & 7 & 7 \\
\hline
\end{tabular}

We also see that USN has the largest number of CVE-IDs in the low range, and lowest number of CVE-IDs in the High range comparing with other VDs in each year.

As mentioned before, it may be that some CVE-IDs do not have a score in a VD, this is represented as "Not available" in table III, and NA in Figures 3 to 6. The NA scores for 2020 are all related to recently published CVE-IDs, and it usually takes some time for the score be updated in VDs. However, USN reported the largest NA scores in 2018 for the vulnerable packages with an unknown impact on Ubuntu releases.

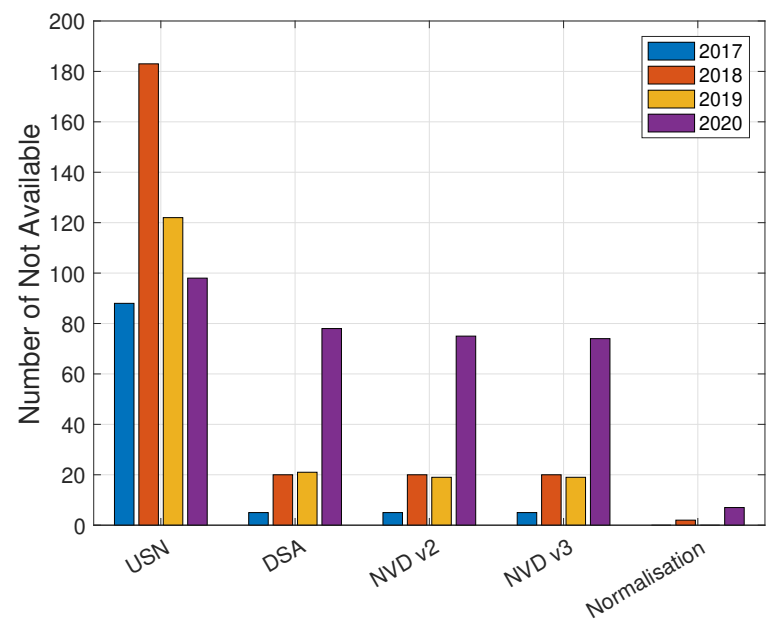

Fig. 7: Distribution of Not Available Score in 2017-2020

\section{Impact of Normalization}

Figures 3 to 6 shows the distribution of the severity score range in VDs and normalisation framework. The normalisation score is distributed in the four ranges Low, Medium, High, and Critical and the number of CVE-ID in the range None is zero in 2017-2020. We also identify the number of CVE-IDs without a score (cf., NA): in 2017 and 2019 it is zero after normalization regardless of the VMM, while the number on CVE-IDs with NA score are two $(0.06 \%)$ and seven $(0.33 \%)$ in 2018 and 2020 relatively. Hence, after normalization the number of the CVE-IDs with not available score is negligible.

We observe the benefits of normalization on the better coverage on CVE-IDs score. Table V presentes the number of CVE-IDs that does not have a score in each VDs and the changes on the score after applying normalization framework. We find the change in the score range for CVE-IDs with NA, does not depend on the VMM. Hence, the result in Table V is the same for Basic, Standard, and Restrictive mode.

We visualized the change in not available score in VDs and normalisation in Figure 7. We noticed the most of the CVE-IDs ranked NA in VDs, moved to the medium range after applying normalization framework.

To have a fair comparison of the normalization impact, we calculate the percentage of the CVE-IDs, which does not change its range after applying the normalization framework. Table IV presents the percentage of the changed and unchanged severity score range in 2017-2020. We noticed that USN reported a higher percentage of unchanged severity score range in the basic VMM, while the restrictive VMM provides the higher unchanged range in NVD and DSA.

\section{CONCLUSION}

We proposed and implemented a normalization framework to facilitate classification and evaluation phases in VRM when dealing with multiple VDs. Our solution provides a numeric security score for each CVE-ID by applying a normalization 
TABLE IV: The percentage of the CVE-ID with changed and unchanged severity score range after normalization 2017-2020

\begin{tabular}{|c|c|c|c|c|c|c|c|c|c|}
\hline \multicolumn{2}{|c|}{ Year } & \multicolumn{2}{c|}{2017} & \multicolumn{2}{c|}{2018} & \multicolumn{2}{c|}{2019} & \multicolumn{2}{c|}{2020} \\
\hline VMM & VD & \% unchanged & \% changed & \% unchanged & \% changed & \% unchanged & \% changed & \% unchanged & \% changed \\
\hline \multirow{3}{*}{ Basic } & USN & 75,2 & 24,8 & 81,5 & 18,5 & 78,4 & 21,6 & 78,2 & 21,8 \\
\cline { 2 - 10 } & DSA & 62,8 & 37,2 & 71,1 & 28,9 & 72 & 28 & 77,5 \\
\cline { 2 - 10 } & NVD v2.0 & 62,8 & 37,2 & 71 & 29 & 72,1 & 27,9 & 7,3 \\
\hline \multirow{3}{*}{ Standard } & USN & 71 & 29 & 79,1 & 20,9 & 75,9 & 24,1 & 77,4 & 22,7 \\
\cline { 2 - 10 } & DSA & 66,9 & 33,1 & 73,4 & 26,6 & 74,7 & 25,3 & 78,3 & 21,7 \\
\cline { 2 - 10 } & NVD v2.0 & 66,9 & 33,1 & 73,3 & 26,7 & 74,8 & 25,2 & 78,1 & 21,9 \\
\hline \multirow{3}{*}{ Restrictive } & USN & 49,2 & 50,8 & 59,5 & 40,5 & 59,5 & 40,5 & 66,3 & 33,7 \\
\cline { 2 - 10 } & DSA & 87,6 & 12,4 & 93,1 & 6,9 & 91,2 & 8,8 & 89,3 & 10,7 \\
\cline { 2 - 10 } & NVD v2.0 & 87,6 & 12,4 & 93 & 7 & 91,3 & 8,7 & 89,1 & 10,9 \\
\hline
\end{tabular}

TABLE V: The change in severity score of Not Available in VDs after normalisation 2017-2020

\begin{tabular}{|c|c|c|c|c|c|c|c|}
\hline Year & VD & Not Available(NA) & $\mathrm{NA} \rightarrow \mathrm{NA}$ & NA $\rightarrow$ Low & NA $\rightarrow$ Medium & NA $\rightarrow$ High & NA $\rightarrow$ Critical \\
\hline \multirow{3}{*}{2017} & USN & 88 & 0 & 4 & 55 & 17 & 12 \\
\hline & DSA & 5 & 0 & 2 & 2 & 1 & 0 \\
\hline & NVD & 5 & 0 & 2 & 2 & 1 & 0 \\
\hline \multirow{3}{*}{2018} & USN & 183 & 2 & 8 & 149 & 21 & 3 \\
\hline & DSA & 20 & 2 & 0 & 18 & 0 & 0 \\
\hline & NVD & 20 & 2 & 0 & 18 & 0 & 0 \\
\hline \multirow{3}{*}{2019} & USN & 122 & 0 & 10 & 90 & 21 & 1 \\
\hline & DSA & 21 & 0 & 4 & 17 & 0 & 0 \\
\hline & NVD & 19 & 0 & 4 & 15 & 0 & 0 \\
\hline \multirow{3}{*}{2020} & USN & 98 & 7 & 18 & 64 & 8 & 1 \\
\hline & DSA & 78 & 7 & 19 & 52 & 0 & 0 \\
\hline & NVD & 75 & 7 & 19 & 49 & 0 & 0 \\
\hline
\end{tabular}

algorithm. The same type of score (i.e., numeric score) from multiple VDs helps the security decision-maker to generalize the severity score. In this study, we evaluated the normalization framework's impact on a large set of sampled CVE-IDs and analyzed the distribution of severity scores.

This study shows the significant impact of the VDs' severity score on classification, evaluation, and patch prioritization in VRM. Hence, selecting one general scoped VD to use in VRM would not be sufficient for patch prioritization as environmental metrics are not considered in the score calculation. Based on our result, we recommend security expert to select at least one subject-specific and one general VD, and to use the normalized scores for decision making in the organizations's VRM.

Our study declares that we need multiple VDs as a reference in VRM to get better severity score coverage of the known CVE-IDs. Our result indicates the organization assets should be used as essential criteria for the selection of reference VDs. For example, suppose the organization used the Dell servers and Debian's operating system. In that case, they have to use Dell's and Debian's VD as reference VDs in VRM.

In the future, we will add another subject-specific VD, such as RedHat, to our sample VDs for expanding our study on the impact of the normalization framework. We also will classify, evaluate and prioritize the patch based on normalized score.

\section{ACKNOWLEDGMENT}

The work of E.Casalicchio is partially funded by the Project SmartDefense: Models, algorithms and mechanisms for Reducing Cyber Risks in Smart Industry, Ricerca Ateneo 2019.

\section{REFERENCES}

[1] A. Amini and N. Jamil, "A comprehensive review of existing risk assessment models in cloud computing," in Journal of Physics: Conference Series, vol. 1018, no. 1. IOP Publishing, 2018, p. 012004.
[2] “NIST National Vulnerability Database,” https://nvd.nist.gov/, [Online; accessed 30-January-2021].

[3] "Ubuntu Security Notice," https://usn.ubuntu.com/, [Online; accessed 8-March-2020].

[4] "Debian Security Tracker," https://www.debian.org/security/\#DSAS, [Online; accessed 29-April-2020].

[5] "RedHat Security Advisories," https://access.redhat.com/security/ security-updates/\#/, [Online; accessed 8-March-2020].

[6] K. Kritikos, K. Magoutis, M. Papoutsakis, and S. Ioannidis, "A survey on vulnerability assessment tools and databases for cloud-based web applications," Array, vol. 3, p. 100011, 2019.

[7] D. Dey, A. Lahiri, and G. Zhang, "Optimal policies for security patch management," INFORMS Journal on Computing, vol. 27, no. 3, pp. $462-477,2015$.

[8] G. L. Guzie, "Vulnerability risk assessment," ARMY RESEARCH LAB WHITE SANDS MISSILE RANGE NM, Tech. Rep., 2000.

[9] "European Cybersecurity Certification Scheme for Cloud Services," https: //www.enisa.europa.eu/publications/eucs-cloud-service-scheme, [Online; accessed 11-February-2021].

[10] W. Jansen and T. Grance, "Sp 800-144. guidelines on security and privacy in public cloud computing," 2011.

[11] M. Hogan, F. Liu, A. Sokol, and J. Tong, "Nist cloud computing standards roadmap," NIST Special Publication, vol. 35, pp. 6-11, 2011.

[12] V. Singh and S. Pandey, "Cloud computing: Vulnerability and threat indications," in Performance Management of Integrated Systems and its Applications in Software Engineering. Springer, 2020, pp. 11-20.

[13] V. Ahmadi, P. Arlos, and E. Casalicchio, "Normalization of severity rating for automated context-aware vulnerability risk management," in 2020 IEEE International Conference on Autonomic Computing and SelfOrganizing Systems Companion (ACSOS-C). IEEE, 2020, pp. 200-205.

[14] "Best Linux distributions for DevOps," https://cloudacademy.com/blog/ linux-and-devops-the-most-suitable-distribution/, [Online; accessed 23January-2021].

[15] "Open Web Application Security Project," https://www.owasp.org, [Online; accessed 07-March-2021]

[16] P. Kamongi, S. Kotikela, K. Kavi, M. Gomathisankaran, and A. Singhal, "Vulcan: Vulnerability assessment framework for cloud computing," in 2013 IEEE 7th International Conference on Software Security and Reliability. IEEE, 2013, pp. 218-226. 
[17] "The State of Open Source Security VULNERABILITIES," https://www.whitesourcesoftware.com/ open-source-vulnerability-management-report/, [Online; accessed 23-January-2021].

[18] M. J. Haber and B. Hibbert, "Vulnerability management architecture," in Asset Attack Vectors. Springer, 2018, pp. 213-216.

[19] M. Walkowski, M. Krakowiak, J. Oko, and S. Sujecki, "Distributed analysis tool for vulnerability prioritization in corporate networks," in 2020 International Conference on Software, Telecommunications and Computer Networks (SoftCOM). IEEE, 2020, pp. 1-6.

[20] "Common Vulnerability Scoring System(CVSS v2.0: User Guide)," https: //www.first.org/cvss/v2/guide, [Online; accessed 2-February-2021].

[21] "Common Vulnerability Scoring System(CVSS v3.0: User Guide)," https: //www.first.org/cvss/v3.0/user-guide, [Online; accessed 2-February-2021] 